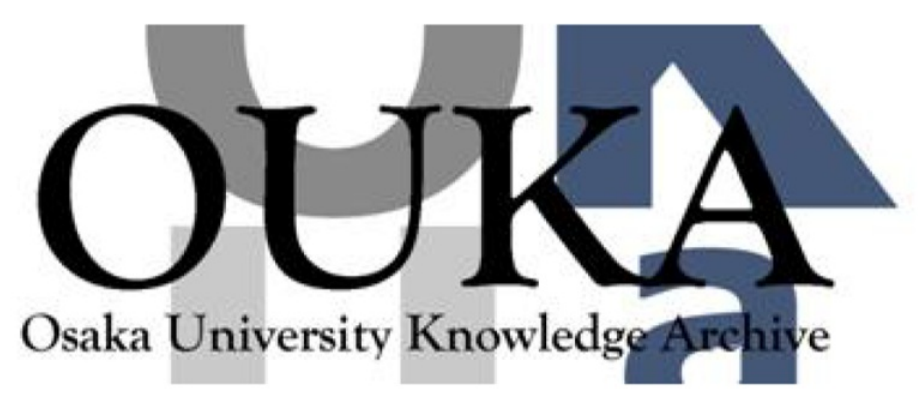

\begin{tabular}{|c|c|}
\hline Title & $\begin{array}{l}\text { Structure and dynamics of suspensions of silica } \\
\text { particles in liquid crystals under a low } \\
\text { frequency ac applied voltage }\end{array}$ \\
\hline Author (s) & Lee, S.B. ; Nakayama, K.; Matsui, T. et al. \\
\hline Citation & $\begin{array}{l}\text { IEEE Transactions on Dielectrics and Electrical } \\
\text { Insulation. } 9(1) \text { p. } 31-\text { p. } 38\end{array}$ \\
\hline Issue Date & $2002-02$ \\
\hline oaire:version & VoR \\
\hline URL & https://hdl. handle. net/11094/3011 \\
\hline rights & $\begin{array}{l}\text { (2002 IEEE. Personal use of this material is } \\
\text { permitted. However, permission to } \\
\text { reprint/republish this material for advertising } \\
\text { or promotional purposes or for creating new } \\
\text { collective works for resale or redistribution } \\
\text { to servers or lists, or to reuse any } \\
\text { copyrighted component of this work in other } \\
\text { works must be obtained from the IEEE. }\end{array}$ \\
\hline Note & \\
\hline
\end{tabular}

Osaka University Knowledge Archive : OUKA

https://ir. Library. osaka-u. ac. jp/

0saka University 


\title{
Structure and Dynamics of Suspensions of Silica Particles in Liquid Crystals under a Low Frequency ac Applied Voltage
}

\author{
S. B. Lee ${ }^{1}$, K. Nakayama, T. Matsui, M. Ozaki and K. Yoshino \\ Department of Electronic Engineering \\ Graduate School of Engineering \\ Osaka University \\ 2-1 Yamada-Oka, Suita, Osaka 565-0871, Japan
}

\begin{abstract}
Silica particles dispersed in liquid crystals exhibit a novel behavior such as a unique migrating behavior and alignment. These characteristics are found to depend on the size of the nanoparticles, the surface state of nanoparticles, the liquid crystal (LC) phases, the amplitude and the frequency of applied voltage. These are discussed in terms of anisotropic particle-particle and particle-LC molecules interactions in the anisotropic environment of liquid crystal matrix.
\end{abstract}

\section{INTRODUCTION}

$\mathrm{S}$ USPENSIONS of small dielectric particles in isotropic dielectric liquids have been studied for a long time [1-5]. If dielectric constants of particles and a liquid are mismatched, the high electric field applied to such dispersions induces long-range dipole interaction between the induced dipole moments of the particles. If the field exceeds a critical value it leads to one-dimensional chaining of particles along field lines [3] and, finally, to columns of particles of a body-centered tetragonal lattice, in which the particles are closed-packed along the electric field direction $[5,6]$.

Recently, dispersions of small dielectric particles in anisotropic dielectric liquids have attracted much attention from fundamental interest as well as because it had a technological potential [7-15]. One example of such dispersion is the so called filled nematic liquid crystal (FNLC) which consists of silica particles in nematic liquid crystal (LC). Fundamental interest includes new type of colloidal anisotropic interactions because of strong elastic distortions of the liquid crystal host which can be useful in the design of new composite materials with potentially useful applications and the formation of a desired structures with controlled ordering at different length scales [7-11].

In anisotropic dielectric liquids at least three additional interparticle interactions come into play [7]: 1. Because of incompatibility between the boundary conditions on particle surface and at infinity the silica particles give rise to topological defects such as disclinations in the director field $(n)$ which results in an effective interparticle interac- tion. This interaction is highly anisotropic and depends on the elastic constants of LC and on the interaction energy between liquid crystal molecules and the particle surfaces (the anchoring strength of $n$ on particles surfaces). Using electrostatic analogy, it has been shown that the long-range attractive force between particles has a dipolar character for normal or homeotropic anchoring and leads to the formation of chainlike structures. The repulsion arises from the presence of a topological defect between neighboring particles. For tangential or homogeneous anchoring, the long-range interaction is theoretically expected to have a quadrupolar character. For example, the qualitative pair particle interaction potential for a weak anchoring is given by $U \propto W^{2} a^{8} / K d^{5} \gg k_{B} T$ [11] where $W$ is the anchoring energy, $a$ the particle size, $K$ elastic constant, $k_{B}$ the Boltzmann constant and $T$ the temperature. This interaction potential is highly anisotropic and depends not only on the inter-particles distance $d$ and the orientation of the line connecting the particles centers relative to director field but also on the anchoring energy $W a / K$ and the particle size $a$. The effective anchoring strength and, consequently, the interaction potential is controlled by the particle size. 2. Attractive interparticle interaction induced by a gradient in the magnitude of the nematic order parameter in the particle neighborhood. 3. Long-range forces are induced by orientational fluctuations in anisotropic mesophases [16]. In nematic LC with symmetrical boundary conditions, this force is $F \propto d^{-3}$. In smectics this force is $\alpha d^{-2}$, and longer in range than van der Waals forces since $K_{2}$ and $K_{3}$ diverge while $K_{1}$ remains finite ( $K_{1}, K_{2}$ and $K_{3}$ are the Frank elastic constants). 
In the case of silica particles, hydrogen bonds between a surface silanol groups of adjacent silica particles and hydrogen bonds between silica particles and liquid crystal molecules should also be taken into account [13].

The potential applications of dispersions of small dielectric particles in anisotropic dielectric liquids comprise a broad spectrum from the possibility of formation of new topologically controlled colloidal structures on different length scales by balancing the attractive and repulsive organizing forces [17] to recently introduced new liquid crystal display technique $[12,13]$.

Interplay of the van der Waals attractive forces and the repulsive hard-core interaction makes the dielectric colloids intrinsically unstable and leads to flocculation. Screened electrostatic interactions govern the structure of stabilized charged colloidal particles in an isotropic dielectric liquid and are quantitatively described with the well established Derjaguin-Landau-Verwey-Overbeek theory [18].

On the other hand, in the case of anisotropic dielectric liquids such as liquid crystals the behavior of silica particles in LC can be viewed in the purely dielectric interaction picture. In liquid crystals of very high purity the ionic current through the sample is negligibly small $\left(<10^{-4}\right.$ $\mathrm{A} / \mathrm{m}^{2}$ ) and no electrohydrodynamic flow was observed in polarizing optical microscope.

In this paper we present the results of study of dynamical behavior of silica particles dispersed in liquid crystals under a low frequency ac applied voltage. Some preliminary results have been published earlier [15]. Silica particles exhibit a novel behavior such as a unique migrating behavior and alignment. These characteristics are found to depend on the size of particles, the surface state of nanoparticles, liquid crystal phases, the amplitude and the frequency of applied voltage.

\section{EXPERIMENT}

We have studied several types of silica particles with particle size $d_{p}$ of $7 \mathrm{~nm}$ (AEROSIL A380) and $40 \mathrm{~nm}$ (AEROSIL OX50) (Degussa-Hüls AG, Germany), $200 \mathrm{~nm}$, $1 \mu \mathrm{m}$ and $3 \mu \mathrm{m}$ (Catalysts \& Chemicals Ind. Co. Ltd., Japan). All particles are non-porous and have no internal surface area. Aerosil nanoparticles were used without any additional treatments as supplied by Degussa Corp.

The nematic LC mixtures BL011 and BL035 (Merck Ltd.) with positive dielectric anisotropy $\left(\Delta \epsilon=\epsilon_{\|}-\epsilon_{\perp}=\right.$ +16.2 for BL011 and +16.6 for BL035 at $1 \mathrm{kHz}$ ) were used as an anisotropic dielectric host liquid. One-hour stirring was used to ensure spatial homogeneity of filled nematic LC mixtures. The concentration of nanoparticles was varied in the range of 3-27 wt.\%. Stable dispersions of very high volume ratio for the nematic LC phase sandwiched between two glass plates with transparent ITO coatings were made for transmission-voltage measure- ment. The sample transmittance $\left(T=I_{\text {out }} / I_{\text {in }}\right)$ was mea"sured using He-Ne laser as a light source and a photodiode for detection of transmitted light. Transmittance-voltage $(T-V)$ dependence of filled nematic cell has been measured upon application of the ac voltage $U=0-170 \mathrm{~V}$ at frequency of $0.01-1 \mathrm{kHz}$. All experiments have been done at relatively low ac voltages to avoid electrical breakdown.

We have also studied the dynamical behavior of individual silica spherical microparticles of $1 \mu \mathrm{m}$ and $3 \mu \mathrm{m}$ in diameter in smectic liquid crystal CS-1024 and CS-1029 (CHISSO Co.) which has the phase sequence of isotropic (Iso)-chiral nematic ( $\mathrm{N}^{*}$ )-smectic A (Sm A)-chiral smectic $\mathrm{C}\left(\mathrm{Sm} \mathrm{C} \mathrm{C}^{*}\right)$. Because of high viscosity of smectic phase the composition was heated up above $\mathrm{N}^{*}$-Iso transition temperature and ultrasonically mixed in isotropic phase. After that the mixture was slowly cooled. The sample was homogeneously aligned between ITO glass plates coated with rubbed polyimide layers. The movement of the microparticles was observed using a polarizing microscope with a CCD camera and recorded by a video recorder.

\section{RESULTS \\ 3.1 SILICA PARTICLES IN NEMATIC LIQUID CRYSTAL}

Figure 1a shows the birefringence patterns around the individual silica particles of $3 \mu \mathrm{m}$ in diameter and its small aggregates in nematic LC sandwiched between two glass plates, coated with rubbed polyimide layer for homogeneous alignment of nematic LC parallel to substrates. The local field at $\mathrm{LC} /$ silica interface favors parallel alignment of nematic LC molecules [19]. The parallel configurations with a pair of accompanying topological surface defects (so called boöjums) at silica particle surface are clearly seen [9]. Particles with such configuration form the characteristic anisotropic aggregates with angle between alignment direction and the line connecting the centers of particles of about $\pm 30^{\circ}[10]$.

Figure $1 \mathrm{~b}$ shows the anomalous long chain-like structure of the same $3 \mu \mathrm{m}$ silica particles in nematic LC formed after addition of small amount of polyoxyethylene sorbitan monolaurate (TWEEN 20). It is known that polyoxyethylene sorbitan monolaurate surfactant can be used to obtain normal anchoring [9]. In this case, the long range dipolar-like attractive interaction leads to chainlike structures. The observed images in Figures $1 \mathrm{a}$ and $1 \mathrm{~b}$ are very similar to those found in inverted emulsions [9] and suspensions of latex particles in nematic LC [10].

TEM micrograph of non-aligned nematic LC filled with $40 \mathrm{~nm}$ hydrophilic AEROSIL nanoparticles (16 wt.\%) in scattering state is shown in Figure 2. After careful mechanical stirring the hydrophilic nanoparticles form a 3-dimensional (3D) sophisticated beadlike structures with liquid crystal domains in empty spaces. Interaction of 

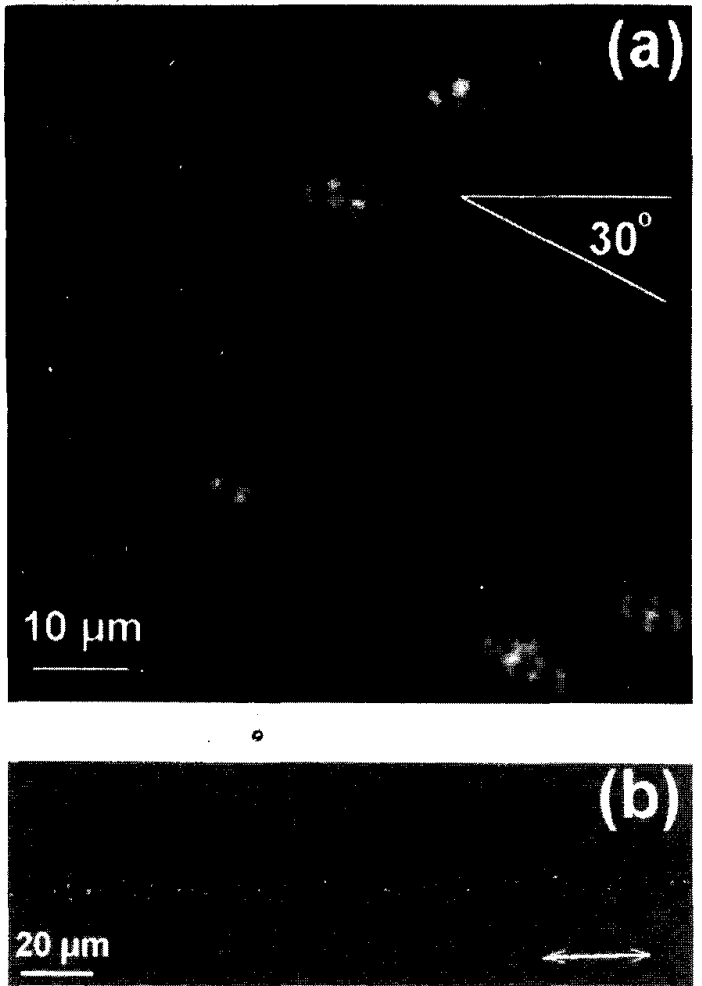

Figure 1. Optical images of silica particles in nematic liquid crystal as was observed using cross polarizers: (a) non-treated particles with parallel anchoring, (b) normal anchoring after treatment with polyoxyethylene sorbitan monolaurate surfactant.

nanoparticles, which are relatively big in comparison with the size of the liquid crystal molecules, can be studied within the continuum approach. In the case of very small

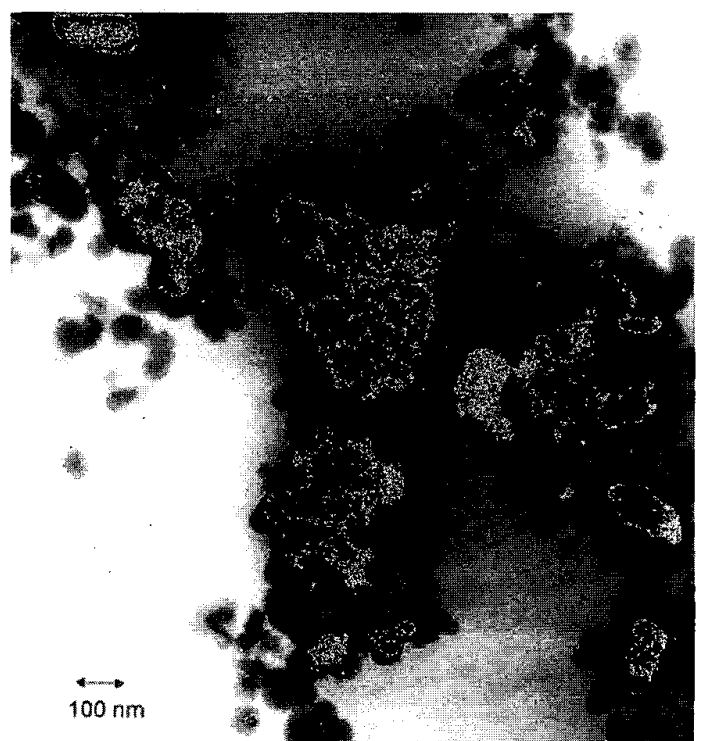

Figure 2. TEM image of nematic LC filled with $40 \mathrm{~nm}$ hydrophilic nanoparticles. nanoparticles $(<10 \mathrm{~nm})$ the strong orientation dependence of induced dipole-dipole type interaction between polarized particles and polar molecules of liquid crystal should be taken into account. However, the effect of elastic distortion on the orientational order is too small. Therefore the repulsive forces is also too small and no special pattern was observed. The characteristic size of particles, beyond which the rigid boundary conditions are valid, is $\tau_{c} \approx K / W$ [20]. Even for the case of very strong anchoring ( $\left.W \approx 10^{-3} \mathrm{~J} / \mathrm{m}^{2}\right)$ we obtain $\tau_{c} \geq 10 \mathrm{~nm}(K \approx$ $10^{-11} \mathrm{~N}$ ). In addition, the thickening effect is relatively limited with $40 \mathrm{~nm}$ particles (AEROSIL OX50) but strongly increases with decrease of particle size [21]. Fractal behavior of colloidal aggregates of small silica nanoparticles in basic solutions was reported [22]. In this case the structure of colloids is strongly dependent on the fine balance of repulsive and attractive forces.

We have further studied the most interesting case of 40 $\mathrm{nm}$ silica nanoparticles in nematic LC. In Figure 3 the temperature dependence of the optical transmittance of nematic LC filled with hydrophilic aerosil nanoparticles OX50 (16 wt. \%-8 vol.\%) is shown. The BL011+ AEROSIL OX50 mixture strongly scatters the visible light in the nematic phase due to large LC birefringence. At $T \geq T_{N I}\left(T_{N I}\right.$ is the nematic-isotropic phase transition temperature) we have observed the sharp change from strong scattering state to weak scattering in the isotropic phase. Therefore, the strong turbidity in nematic phase is not because of multiple scattering by particles aggregates. Strong scattering of visible light was observed in the nematic phase due to large optical anisotropy of LC ( $\Delta n=$ 0.2670 at $589 \mathrm{~nm},+20^{\circ} \mathrm{C}$ ). We did not find the optical hysteresis when light transmittance was measured as a function of temperature. We have also observed the small but clear positive shift of $T_{N I}$ with the increase of concentration of aerosil nanoparticles in the range of 16-24 wt.\%. From the shift of $T_{N I}$ with the increase of concentration of aerosil nanoparticles we obtained very reasonable value for the mean LC domain size of about $0.17 \mu \mathrm{m}$ [23].

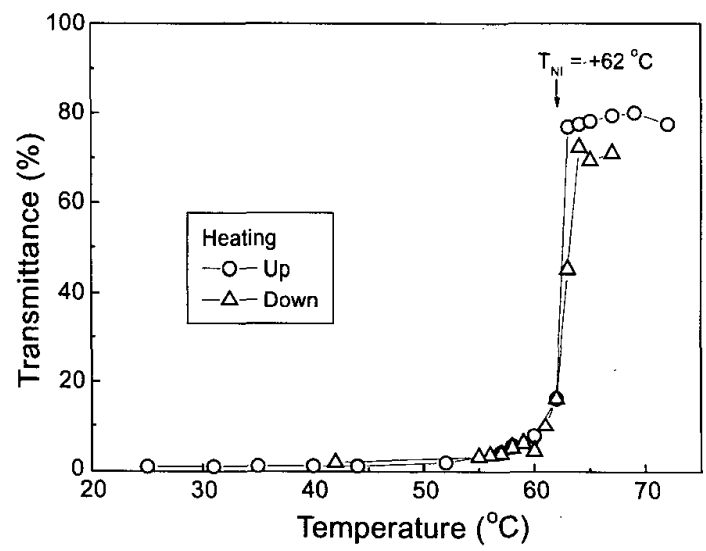

Figure 3. Transmittance-temperature dependence of nematic LC filled with $40 \mathrm{~nm}$ hydrophilic silica nanoparticles ( $\Delta$ up, $\mathrm{O}$ down). 


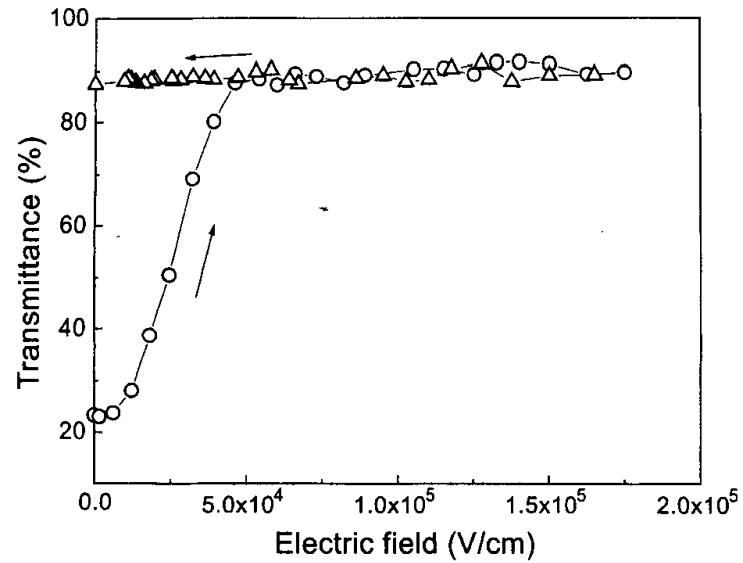

Figure 4. Transmittance-Voltage dependence of nematic LC filled with $40 \mathrm{~nm}$ hydrophilic silica nanoparticles.

Upon application of ac electric field the dispersion has become transparent at threshold voltage of about $20 \sim 30 \mathrm{~V}$ (which correspond to electric field of $2.5 \times 10^{6}-3.8 \times 10^{6}$ $\mathrm{V} / \mathrm{m}$ ) at the particle concentration of $10-18 \mathrm{wt} \%$ (Figure 4). The strong memory effect ( $M \approx 95 \%$ ) was observed in nematic LC filled with relatively big hydrophilic nanoparticles ( $d=40 \mathrm{~nm}, \mathrm{OX} 50)$. The memory parameter $(M)$ was calculated from the ratio $M=\left(T_{r}-T_{o}\right) /\left(T_{s}-T_{o}\right)$, where $T_{o}$ is the initial transmittance, $T_{r}$ the remained transmittance and $T_{s}$ the saturated transmittance. There is a threshold voltage for memory effect. The threshold voltage for memory effect is about $50-100 \mathrm{~V}$ depending on concentration of particles. If the electric field does not overcome the threshold value, the memory parameter would be lower and the switching process is almost reversible. Very small memory effect was observed at first scanning in the mixture with AE380. The memory parameter is about $21 \%$. But after first scanning the $T-V$ curve can be repeatedly measured without hysteresis.

\subsection{MICROPARTICLES IN SMECTIC LIQUID CRYSTAL}

The smectic liquid crystal CS-1024 (CHISSO Co.) has the phase sequence of isotropic (Iso)-chiral nematic $\left(\mathrm{N}^{*}\right)$-smectic A ( $\mathrm{Sm} \mathrm{A}$ )-chiral smectic C ( $\left.\mathrm{Sm} \mathrm{C} \mathrm{C}^{*}\right)$. The study of the typical traces of dielectric particles moving in this LC, confined between two ITO-coated glass plates with rubbed polyimide layers (the cell gap is $12 \mu$ ) under the ac applied voltage of rectangular shape $( \pm 100 \mathrm{~V}$ at 60 $\mathrm{Hz}$ ), has shown the movement of individual particles in all phases. The separate particles migrate linearly in both the layered $\mathrm{Sm} \mathrm{A}$ and $\mathrm{Sm} \mathrm{C*}$ phases along the smectic layer as shown in Figure 5. The threshold voltage for particle movement depends on the waveform of applied voltage and was found to be about $50 \mathrm{~V}$ for symmetrical square waveform. Such movement of particles was not observed at $\mathrm{dc}$ and asymmetric monopolar voltages.
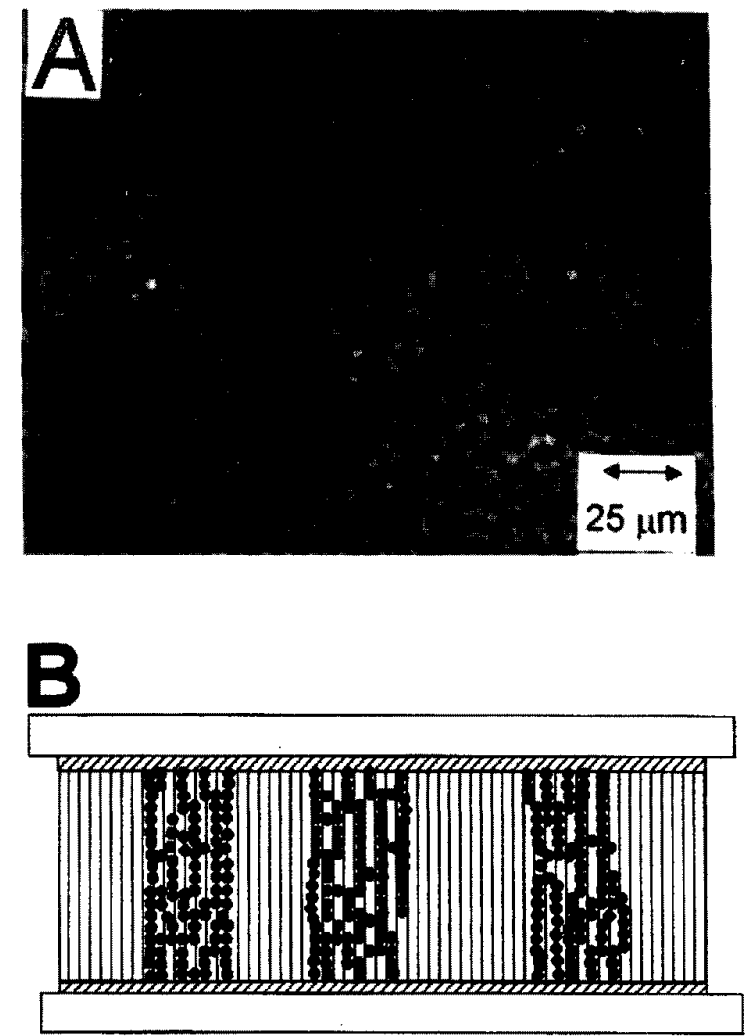

Figure 5. (a) Optical micrograph of agglomerates of $40 \mathrm{~nm}$ silica nanoparticles in nematic LC after application of ac electric field. (b) Schematic drawing of cross-section view of filled nematic LC in memory state.

Figure 6 shows two examples of regular agglomerates with hexagonal packing of $3 \mu \mathrm{m}$ particles modified by surfactant in smectic $\mathrm{C}^{*} \mathrm{LC}(\mathrm{CS}-1029)$. The arrow indicates

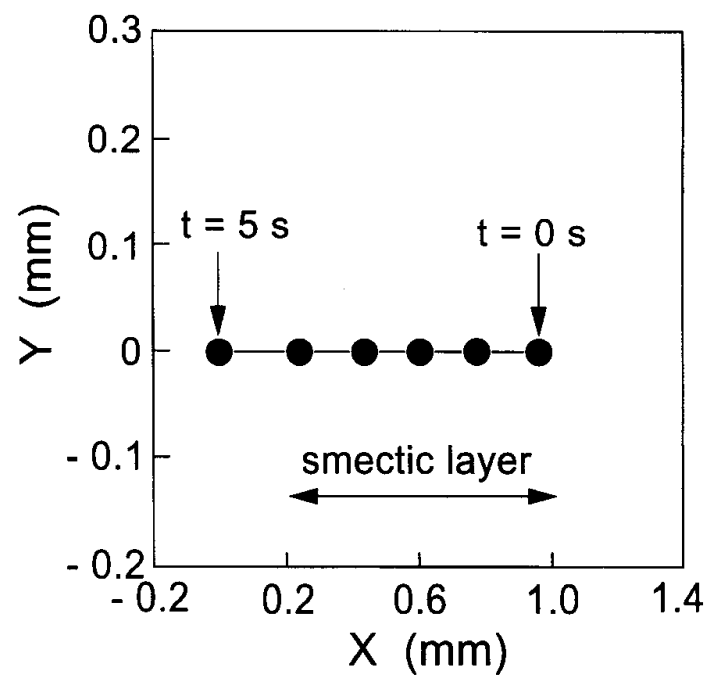

Figure 6. Typical trace of the moved $1 \mu \mathrm{m}$ silica particles in $\mathrm{Sm} \mathrm{C}^{*}$ phase. Rectangular waveform of the voltage $( \pm 100 \mathrm{~V}, 60 \mathrm{~Hz})$ was applied. Each point was plotted for $5 \mathrm{~s}$ at $1 \mathrm{~s}$ interval. the two-sided arrow indicates the direction parallel to smectic layers. 
the direction of smectic layers. Such big agglomerate of particles can be destroyed only at very high electric field ( $\geq 10^{7} \mathrm{~V} / \mathrm{m}$ ) when the current become sufficient to induce the strong flow effects.

\section{DISCUSSION}

\subsection{SILICA PARTICLES IN NEMATIC LIQUID CRYSTAL}

In anisotropic dielectric liquid new anisotropic interactions arise between dielectric silica particles even without applied electric field, resulting in 3D beadlike structures of particles connected by weak hydrogen bonds. It is known that the formation of hydrogen bonds occurs between a surface silanol groups of adjacent AEROSIL particles which leads to an enhanced thixotropy in the nonpolar and semi-polar systems dispersed with AEROSIL nanoparticles. The formation of weak hydrogen bonds between particle-particle and particle-LC in filled nematic LC has been confirmed experimentally by IR spectroscopy [24]. Near homeotropic orientation of 5CB dimers was changed to a chaotic one upon addition of aerosil nanoparticles due to near planar orientation of LC molecules bonded to aerosil surface. However, the hydrogen bonds were not observed in the LC mixed with the particles modified by the hydrofobic molecular fragments of polymethylsyloxane.

As was noted by Ruhwandl and Terentjev [11], the effect of external electric or magnetic field has negative effect on such particle chaining at low concentration of particles because the orientational order becomes more rigid (it decays exponentially as $\sim \frac{1}{r} e^{-r / \xi_{H}}, \xi_{H}$ the magnetic coherence length), and the long-range attractive potential between particles becomes shorter in range. Therefore, the liquid crystalline colloids should behave similarly to typical electrorheological liquids upon application of dc or low frequency ac electric field.

The electric field induces the dipole moment in dielectric spherical particle

$$
\mu=\epsilon_{f} a^{3} \frac{\epsilon_{p}-\epsilon_{f}}{\epsilon_{p}+2 \epsilon_{f}} E_{l o c}
$$

and leads to a long-range attractive potential between two spheres

$$
U=\frac{\mu^{2}}{\epsilon_{f} r_{i j}^{3}}\left(1-3 \cos ^{2} \theta_{i j}\right)
$$

where $\epsilon_{f}$ and $\epsilon_{p}$ are the relative dielectric constants of the liquid and the particle, respectively, $a$ is the radius of particle, $E_{l o c}$ the local electric field, $\theta_{i j}$ the angle between the line connecting the centers of particles and the external field, and $r_{i j}$ the separation between sphere's centers.
In typical electrorheological liquids the dielectric particles with high dielectric constant are dispersed in an isotropic dielectric liquid with a low dielectric constant $\left(\epsilon_{p}\right.$ $\left.-\epsilon_{f}>0\right)$. In the case of silica particles $\left(\epsilon_{p} \approx 4\right)$ dispersed in nematic liquid crystal (for example, $\epsilon_{f \|}=22$ and $\epsilon_{f \perp}=$ 5.4 for BL035) we have the case of negative dielectric contrast $\left(\epsilon_{p}-\epsilon_{f}<0\right)$. For both cases, the interaction between particles is attractive and results in chaining. Then the phase separation occurs in longer time range because of the formation of close-packed columns of particles due to attractive interaction between particle's chains induced by the strong one-dimensional Landau-Peierls thermal fluctuations on dipole chains. The effect of such thermal fluctuations can be taken into account as an effective increase of the Hamaker constant [3]. Increase of the difference between dielectric constants of particles and liquid enhances the interaction potential. Hence, the strong positive dielectric anisotropy $\left(\Delta \epsilon=\epsilon_{\|}-\epsilon_{\perp}>0\right)$ of LC favors the chaining effect along the direction of electric field $(\Delta \epsilon$ $=+16.2$ for BL011 and $\Delta \epsilon=+16.6$ for BL035 at $1 \mathrm{kHz}$ ). The orientational elastic forces of LC further enhances the lining effect.

At high enough electric field the 3D bead-like structure of silica particles transforms into linear chains between electrodes and then collapsed to separate agglomerates of more closely-packed columns of particles. The formation of hydrogen bonds between particles is reversible. It can be broken by shear and readily formed again when the shear stress is removed. Re-orientational forces of liquid crystal break the hydrogen bonds both between the aerosil nanoparticles and between the aerosil particles and LC molecules during the electric poling process. The network of silica nanoparticles is reorganized to a new one with new hydrogen bonds and new internal interfaces and stabilizes the homeotropic alignment after removal of electric field [25]. The torque exerted on particle $(\Gamma \approx$ $(K \Delta \Theta / L) \times S$ where $K$ is a typical nematic elastic constant, $L-$ a half of LC domain size, $\Delta \Theta$ the angle between director in the middle of LC domain and at the surface of particle and $S$ the area of particle) is comparable with the typical energy of hydrogen bonds of $\Delta \mathrm{G} \approx 10-40 \mathrm{~kJ} / \mathrm{mol}$ $\approx 10^{-20} \mathrm{~J} /$ bond [13]. The important thing is, as was noted by Krauzer et al. [13], that the broken hydrogen bonds are formed again, so the energy required for reorganization of the system is less than the energy required for complete breaking of hydrogen bonds.

These agglomerates are invisible in the optical microscope due to memory effect. In nematic LC the surfaces can exert their influence to distances up to several thousands of angstroms. But these agglomerates can be made visible due to distortion of LC alignment around agglomerates by short heating at temperatures $\leq T_{N I}$ (Figure 7a). Thus, the peculiarity of FNLC system with silica nanoparticles is its behavior under influence of electric field. The micron-size agglomerate with aligning sur- 

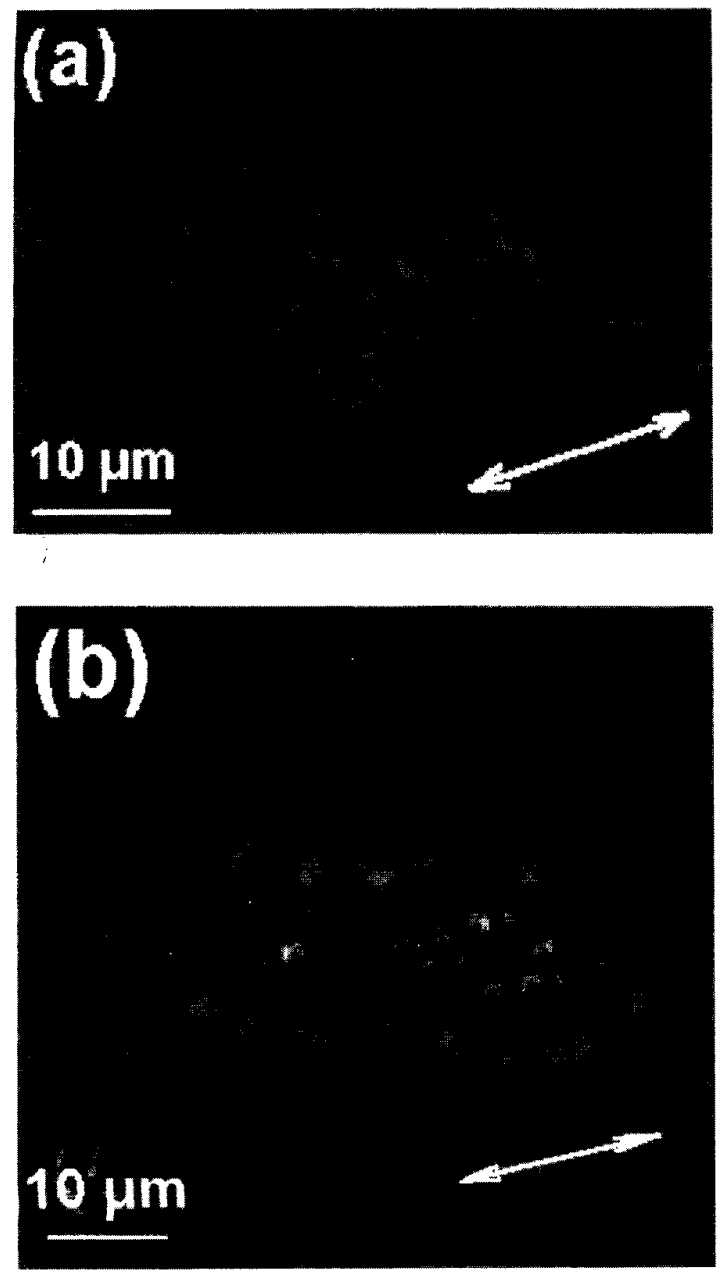

Figure 7. Two examples of regular patterns of $3 \mu \mathrm{m}$ silica particle modified by surfactant, formed in smectic $\mathrm{C}^{*}$ liquid crystal. The white arrow indicates the direction parallel to smectic layers.

faces are formed rather than a continuous $3 \mathrm{D}$ network (Figure $7 \mathrm{~b}$ ). The attractive force between particle chains $\left(F_{\mathrm{attr}} \propto \frac{\sqrt{k_{B} T} \beta E R^{3 / 5}}{r^{3}}\right.$, where $R$ is the diameter of chain or column and $r$ the distance between chains [4]) is opposed by short range perpendicular repulsive elastic forces. These anisotropic interactions prevent the collapse of particle's chain into more close-packed structure and the suspension remains in the transparent state.

The threshold voltage for the memory effect corresponds to the energy of breaking up the hydrogen bonds both between the aerosil particles and between the aerosil particles and LC during the electric poling process due to orientational forces of liquid crystal and the energy required for particle chaining. Upon increasing the electric field the existing aerosil network was broken up, the new system of micron-size agglomerates with aligning surfaces was formed and the optical transmittance curve went to saturation. This transparent state was memorized because the new system of micron-size agglomerates with aligning surfaces is stable and retains after turning off the electric field.

We used the UV-curable photopolymer (5wt.\%) to limit the movement of particles by formation of a polymer network before electric field was applied to FNLC mixture. The memory effect was diminished to a very small value $<1 \%$. Hence, the movement of particles and the reorganization of particles structure is important for memory effect.

\subsection{MICROPARTICLES IN SMECTIC LIQUID CRYSTAL}

In the previous section we discussed the silica particles in nematic LC with a strong positive dielectric anisotropy $(\Delta \epsilon \approx+16)$. Silica particles in smectic liquid crystal with layered structure and the negative dielectric anisotropy present another interesting system. We used the commercially available smectic liquid crystals CS-1024 and CS1029 (CHISSO Co.) which have the phase sequence of isotropic (Iso)-chiral nematic $\left(\mathrm{N}^{*}\right)$-smectic A (Sm A)-chiral smectic $\mathrm{C}\left(\mathrm{Sm} \mathrm{C} \mathrm{C}^{*}\right)$. The effect of long range positional ordering of smectic LC in the form of layered structures on particle behavior is very interesting.

It is known that the impurity ions easily move along the smectic layers. For example, it has been shown that the mobility of ions in ( $p$ - $n$-butoxybenzilidene- $p$-octylaniline) parallel to the smectic layer is much larger than that perpendicular to the smectic layer [26]. The movement of big micron-size particles also occurs preferentially along smectic layers.

Of three possible elastic distortions in liquid crystals, in smectic LC the bend and twist are prohibited. The corresponding elastic constants $K_{2}$ and $K_{3}$ diverge near nematic-smectic phase transition temperature. The movement of particles in smectic LC involves extremely high distortion energy. It should also be mentioned that in the smectic phase the viscosity perpendicular to the layer is extremely high.

At this stage, the origin of movement of particles in layered smectic phase is not clear. The purity of the host liquid crystal was high. Consequently, the driving force of particle movement should not be the ionic flow and might be the dielectric force originating from the interface of the composites. The movement of individual particle was observed in all phases and, therefore, not associated with ferroelectric features in Sm $\mathrm{C}^{*}$ phase, such as a molecular reorientation on the tilt cone at a polarization reversal. The threshold voltage of particle movement depends on the waveform of applied voltage and was found to be about $50 \mathrm{~V}$ for symmetrical square waveform. We should mention that the dynamics of particles strongly depend on the geometry of the cell. The helical pitch size of CS-1024 and 
CS-1029 in the chiral smectic phase was $>20 \mu \mathrm{m}$ and 2 $\mu \mathrm{m}$, correspondingly. But strong electrical field unwinds the helix.

It is not easy to completely avoid the effect of impurity ions in smectic LC, especially at high electric field. The presence of some impurity ions and the negative anisotropy of dielectric constants can cause the asymmetry between polarization of particles and the direction of applied field. The movement of particles, observed only after application of rectangular symmetric ac electric field, can be explained from the perspective of a Fourier transform. The Fourier transform of a square wave signal comprises the high frequency components acting after every polarity change. This explains why the polarity reversal of the applied voltage is necessary. The movement of particles was not observed at $\mathrm{dc}$ and asymmetric monopolar voltages.

The behavior of particles in smectic $\mathrm{C}^{*} \mathrm{LC}$ at high concentration is very different from nematic $\mathrm{LC}$ with positive dielectric anisotropy. In the case of smectic C $\mathrm{C}^{*} \mathrm{LC} \epsilon_{\|} \approx \epsilon_{p}$ $\left(\epsilon_{\|}=2.9\right.$ and $\epsilon_{\perp}=22.2$ at $1 \mathrm{kHz}$ and $40^{\circ} \mathrm{C}$ for CS-1029, while $\left.\epsilon_{p} \approx 4\right)$. Hence, there is no long range induced dipole-dipole attractive interaction along the direction of electric field. In this case, the effect of strong electric field is to unwind the helical pitch and impose the rigid long range positional order.

The case of particles treated with hydrophilic surfactant (TWEEN 20) will be discussed here. We have found that the application of strong electric field leads to aggregation of particles and phase separation. That is, when particles are confined between electrodes with spacing $d \geq 2 d_{p}$, the particles are ordered along smectic layers direction (Figure 7). The effect of dynamic coupling between wetting and phase separation in binary liquid mixture with glass particles on pattern evolution was studied by Tanaka et al. [27]. They have found that if there is the difference in wettability of mobile glass particles in two phases, the particles were included in the more wettable phase and formed the ordered pattern at high concentration. In our case, the application of electric field induces the movement of particles and upon collision the particles are attracted by the wetting-layer induced interaction. What is important in the case of silica particles in smectic $L C$ is that in confined geometry the layered structure of smectic phase imposes the positional order on particles aggregates. This result is surprising because, at first glance, it would be thought that such agglomerate should destroy the smectic order. We should note here that such phase separation was also observed in nematic LC but without special orientation of particles agglomerates. Recently, the $2 \mathrm{D}$ colloids subjected to a $1 \mathrm{D}$ periodic potential have been studied both experimentally and theoretically [28,29]. Rich phase behavior was found. We think the term "floating smectic" can be used for such ordered agglomerate. The structure of boundary layer between the smectic liquid crystal and the wetting phase with particles is now under study.

Such system could be very attractive for study of the influence of tunable interface potential on $2 \mathrm{D}$ colloidal crystals. The helical pitch size of smectic liquid crystal are not fixed and can be tuned by temperature or external potential. Hence, the study of effects of commensurate/ incommensurate surface potential seems to be possible. This also opens the possibility of formation of micron-size photonic crystal structures from sub-micron size particles and seems to be very promising in the light of the predicted anisotropic and tunable liquid crystal photonic band gap materials $[30,31]$.

\section{CONCLUSIONS}

$I^{1}$ $\mathrm{N}$ anisotropic dielectric liquid a new anisotropic interactions arise between dielectric silica particles without applied electric field resulting in 3D bead-like structures of particles connected by weak hydrogen bonds. The ac electric field changes the long range anisotropic interaction potential to short range one and leads to agglomeration of particles.

The particle size effect can explain the large memory effect in nematic liquid crystals filled with relatively large $40 \mathrm{~nm}$ nanoparticles. Upon application of ac electric field to the FNLC mixture confined between the two ITO coated glass substrates, nanoparticles are organized to large micron-sized agglomerates of different shapes with oriented internal interfaces due to orientational elastic forces.

New type of colloidal anisotropic interactions can be useful in the design of new topologically controlled colloidal structures with potentially useful applications and formation of desired structures with controlled ordering at different length scales by delicate balancing between the attractive and repulsive colloidal interaction. More complex patterns of desired structure can be made by using special electrode configurations and fixed by photopolymer. This principle could be used in future nanoarchitecture with nanoparticles as building blocks and orientational elasticity of liquid crystal as building force.

The dispersions of particles in layered smectic liquid crystals. is very interesting. This study has shown the possibility of formation of regular structures with positional order during phase separation. The possibility for control of the shape of ordered aggregates and formation of tunable photonic crystal utilizing silica particles dispersed in liquid crystal are now under study.

We have to note that the dynamics of particles in nematic and, especially, in smectic LC is complicated and further elaborate studies are needed. We have only given a simplified analysis in pure dielectric approximation without taking into account the effects of temperature, 
hydrodynamic interactions, osmotic effects and many other features.

\section{ACKNOWLEDGMENTS}

This work was financially supported by the Research for the Future Program of the Japan Society for the Promotion of Science (Project No. JSPS-RFTF96P00206). The work was also supported by a Grant-in-Aid for Scientific Research from Ministry of Education, Culture, Sports, Science and Technology of Japan.

\section{REFERENCES}

[1] W: Winslow, "Induced Fibration of Suspensions," J. Appl. Phys., Vol. 20, pp. 1137-1140, 1949.

[2] S. Fraden, A. J. Hurd, and R. B. Meyer, "Electric-Field-In" duced Association of Colloidal Particles," Phys. Rev. Lett., Vol. 63, pp. 2373-2376, 1989.

[3] J. E. Martin, J. Odinck and T. C. Halsey, "Evolution of Structure in a Quiescent Electrorheological fluid," Phys. Rev. Lett., Vol. 69, pp. 1524-1527, 1992.

[4] L. Landau and E.M. Lifshitz, Statistical Physics, 2nd Ed., Pergamon, Oxford, 1984.

[5] R. Tao and J. M. Sun, "Three-dimensional Structure of Induced Electrorheological Solid,” Phys. Rev. Lett., Vol. 67, pp. 398-401, 1991.

[6] L. C. Davis, "Ground State of an Electrorheological Fluid,"Phys. Rev. A, Vol. 46, pp. R719-R721, 1992.

[7] S. Ramaswamy, R. Nityananda, V. A. Raghunathan and J. Prost, "Power-Law Forces between Particles in a Nematic," Mol. Cryst. Liq. Cryst., Vol. 288, pp.175-180, 1996.

[8] P. Poulin, H. Stark, T. C. Lubensky and D. A. Weitz, "Novel Colloidal Interactions in Anisotropic Fluids," Science, Vol. 275, pp. 1770-1773, 1997.

[9] P. Poulin and D. A. Weitz, "Inverted and Multiple Nematic Emulsions," Phys. Rev. E, Vol. 57, pp. 626-637, 1998.

[10] P. Poulin, N. Frances and O. Mondain-Monval, "Suspensions of Spherical Particles in Nematic Solutions of Disks and Rods," Phys. Rev. E, Vol. 59, pp. 4384-4387, 1999.

[11] R. W. Ruhwandl and E. M. Terentjev, "Long-Range Forces and Aggregation of Colloidal Particles in a Nematic Liquid Crystal," Phys. Rev. E, Vol. 55, pp. 2958-2961, 1997.

[12] R. Eidenschink and W. H. De Jeu, "Static scattering in filled nematic: new liquid crystal display technique," Electronics Lett., Vol. 27, No.13, pp. 1195-1196, 1997.

[13] M. Krauzer, T. Tschudi, W. H. De Jeu and R. Eidenschink, "New Liquid Crystal Display with Bistability and Selective Erasure Using Scattering in Filled Nematics,"Appl. Phys. Lett., Vol. 62, pp. 1712-1714, 1993.

[14] T. Togo, K. Nakayama, M. Ozaki and K. Yoshino, "Electric Field-Induced Migration of $\mathrm{SiO}_{2}$ particles in Smectic Liquid Crystal," Jpn. J. Appl. Phys., Vol. 36, pp. L1520-L1522, 1997.
[15] S. Lee and C. Park, "Agglomeration of Silica Nanoparticles in Filled Nematic Liquid Crystals," Mol.Cryst.Liq.Cryst., Vol. 333, pp.123-134, 1999.

[16] A. Ajdari, L. Peliti and J. Prost, "Fluctuation-Induced LongRange Forces in Liquid Crystals," Phys. Rev. Lett., Vol. 66, pp. 1481-1484, 1991.

[17] M. Muthukumar, C. K. Ober and E. L. Thomas, "Competing Interactions and Levels of Ordering in Self-Organizing Polymeric Materials," Science, Vol. 277, pp. 1225-1227, 1997.

[18] E. J. W. Verwey and J. Th. G. Overbeek, Theory of Stability of Lyophobic Colloids, Elsevier, Amsterdam, 1948.

[19] X. Zhuang, D. Wilk, L. Marucci and Y. R. Shen, "Orientation of Amphiphilic Molecules on Polar Substrates," Phys. Rev. Lett., Vol. 75, pp. 2144-2147, 1995.

[20] T. C. Lubensky, D. Pettey, N. Currier and H. Stark, "Topological Defects and Interactions in Nematic Emulsions," Phys. Rev. E, Vol. 57, pp. 610-625, 1998.

[21] Datasheet of Degussa-Hüls AG, Germany.

[22] D. W. Schaefer, J. E. Martin, P. Wiltzius and D. S. Cannell, "Fractal Geometry of Colloidal Aggregates," Phys. Rev. Lett., Vol. 52, pp. 2371-2374, 1984.

[23] F.M. Aliev and M.N. Breganov, Zh. Eksp. Teor. Fiz., Vol. 95, pp.122, 1989 (Sov. Phys. JETP, Vol. 68, pp. 70, 1989).

[24] G. A. Puchkovskaya, Yu. A. Reznikov, O. V. Yaroshchuk, A. V. Glushchenko, A. A. Yakubov and N. P. Kharchenko, "Vibration Spectra of Liquid Crystal-Aerosil System," SPIE, Vol. 2795, pp. 106-113, 1996.

[25] M. Krauzer, T. Tschudi and R. Eidenschink, "Erasable Optical Storage in Bistable Liquid Crystal Cells," Mol. Cryst. Liq. Cryst., Vol. 223, pp. 219-227, 1992.

[26] K. Yoshino, N. Tanaka and Y. Inuishi, "Anomalous Carrier Mobility in Smectic Liquid Crystal," Jpn. J. Appl. Phys., Vol. 15, pp. 735-736, 1976.

[27] H. Tanaka, A. J. Lovinger and D. D. Davis, "Pattern Evolution Caused by Dynamic Coupling between Wetting and Phase Separation in Binary Liquid Mixture Containing Glass Particles," Phys. Rev. Lett., Vol. 72, pp. 2581-2584, 194.

[28] Q.-H. Wei, C. Bechinger, D. Rudhardt and P. Leiderer, "Experimental Study of Laser-Induced Melting in Two-Dimensional Colloids," Phys. Rev. Lett., Vol. 81, pp. 2606-2609, 1998.

[29] E. Frey, D. R. Nelson and L. Radzihovsky, "Light-Induced Melting of Colloidal Crystals in Two Dimensions," Phys. Rev. Lett., Vol. 83, pp. 2977-2980, 1999.

[30] K. Yoshino and K. Kawagishi, "Photonic Crystal and Its Preparation, Properties and Applications," J. Elect. Mat. Eng., Vol. 7, pp. 2-20, 1998 (in Japanese).

[31] K. Busch and S. John, "Liquid Crystal Photonic Band Gap Materials," Phys. Rev. Lett., Vol. 83, pp. 967-970, 1999.

This paper is based on a presentation given at the 13th International Conference on Dielectric Liquids (ICDL 99), Nara, Japan, 20-25 July 1999.

${ }^{J}$ On leave from Heat Physics Department of Uzbek Academy of Science, Tashkent,Uzbekistan; e-mail:sergey@ele.eng.osaka-u.ac.jp.

Manuscript received 19 September 1999, in final form 30 October 2001. 